\title{
The Silent and Looming — The Visual Schema and Metaphorical Construction of Contemporary Abstract Painting
}

\author{
Zhijun Wang,
}

\author{
${ }^{1}$ Academy of Fine Arts, Shanxi University, Taiyuan, Shanxi 030006, China \\ *Corresponding author. Email: duchy777@163.com
}

\begin{abstract}
The language of abstract painting presents a relationship between silence and loom as well as different narrative methods of abstract painting artists. It corresponds and observes with the artist's own inner world through visual means to achieve the agreement between the meaning and content of the visual form. The abstract works of different artists have a variety of presentation methods in terms of schematic features and work forms. This diversity characterizes the semantic features and linguistic meanings that appear in the visual schema, adding to the abstract metaphorical meaning. The abstract or hidden visual symbols, or the patterns composed of silent or high-profile visual elements in abstract paintings provide people with a way to understand the works. The visual schema formed by the visual elements in the abstract context is read and understood, presenting the changing qualities of artistic image language.
\end{abstract}

Keywords: metaphor, schema transfer, abstract painting, visual logic

\section{INTRODUCTION}

In contemporary art, there has been uninterrupted discussion and attention to abstract painting, and a group of explorers, practitioners, critics and masters of abstract painting have been active and emerging. Although the times and lifestyles people live in are full of new media and digital media, and even have a huge impact on people's visual habits and life content, they still can't replace the diversity of contemporary art practice and the unique status of abstract painting. And in the visual image composed of many new media technologies and cultural practices, people also need to clarify the logic: What is the core and essence of the image? What kind of internalized spirit is the interpretation of images interpreted and appreciated by people? What meanings and representations are contained in and emerge from the visual forms in abstract paintings and visual objects that do not seem to be premised on real realistic images?

\section{LANGUAGE TURN AND NARRATIVE LOGIC}

When people turn to figurative painting, the information and content of the image will point to clarity in understanding and viewing. The understanding of images depends on the things and events they refer to. This kind of viewing and reading is completely different from the formal perception of abstract painting. Greenberg believes that "the meaning of an image is to use art to tell what an image is actually", and "in the context of asking about the essence of the image and what the image is perceived, the so-called 'abstract' image proved to be the most specific and typical image situation".[1] That is to say, in a specific context, the perception and interpretation of abstract painting are closer to and correspond to people's intuition and actions than figurative painting. However, abstract language is often constructed in a relationship between silence and loom, presents different narrative methods of abstract painting artists, is devoted to expressing how abstract paintings can catch up with specific objects visually, and is corresponding to the artist's own inner world so as to achieve a match between the meaning and content of the visual form.

Abstract paintings don't contain more or less realistic representations and descriptions of concrete objects like many realistic paintings and expressive paintings. The visual elements in abstract paintings may be color blocks, interweaving of lines, or many less specific synthesis of blurred visual elements "Works are never finite things, but interactions and transformations within the realm of consciousness".[2] The color of abstract painting may not point to a specific object, but it often corresponds to and presents the artist's emotions, thoughts, and thinking logic. This invisibility, which is different from figurative painting, is clearly displayed in the picture space of abstract painting 
through a corresponding visual language. Standing in front of abstract painting, the schema in abstraction on the one hand acts on the meaning of the painting itself. On the other hand, as the visual element of the picture, the schema in abstraction forms a form of logic and language construction with the space in which it is located. Even in the picture, outside the picture, the schema is linked into multiple interwoven running through, superimposed and looming. The painting opens the metaphorical space under the visual plane through the image itself, and triggers reference and reflection on the meaning of the image.

\section{VISUAL METAPHOR AND PERCEPTION CONSTRUCTION}

The language correspondence between abstract painting and image, space, silence and looming can be interpreted through Tan Ping's 2016 works in the Helmhaus Art Museum in Switzerland, "Mountains Beyond Mountains" ("Fig. 1"). In his works, Mr. Tan Ping not only retained the traces of time that appeared in the previous covering behavior, but also enhanced the narrative features of the work through the interweaving of blocks, lines and colors in the painting: on the one hand, some picture elements are hidden, eliminated and superimposed; on the other hand, more new elements and new narratives are covered and superimposed, presented and accepted, constructing a richer semantic and metaphorical nature. "Typical metaphors involve not only changes in scope, but also changes in domains".[3] This feature of schema transfer continued into Tan Ping's solo exhibition "Stitching and Regeneration: From Zurich to Basel Hong Kong" at the 2018 Hong Kong Basel Art Fair Insights Asian Vision Unit. The lines and shapes in the picture of the work began to "overflow" to the wall of the exhibition hall, expanding the new spatial vision and dialogue relationship. This dialogue relationship exists not only between the content overflowing inside and outside the screen, but also in the multiple contexts formed by the works people and the exhibition space, forming the viewing logic and schema outside the field and in the vision and giving birth to a rich and connotative metaphorical spiritual realization.

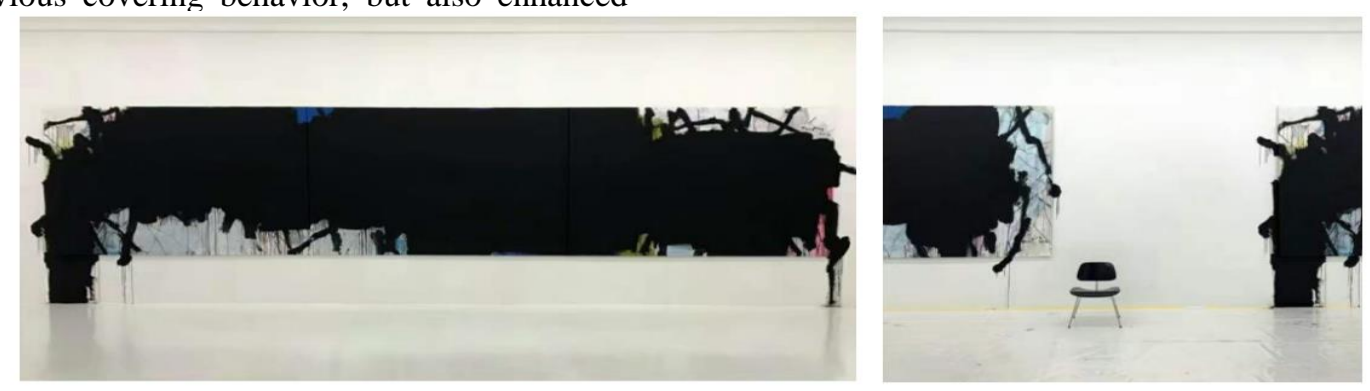

Fig. 1. Tan Ping's 2016 exhibition work of "Mountains Beyond Mountains" at Helmhaus Art Museum in Switzerland.

As a leading figure in contemporary Chinese abstract painting, many of Tan Ping's painting languages are implicit, silent and expressive. People can feel the power of thinking from the shape of these thoughts. The subtlety and silence in this kind of painting are different from the action painting of the abstract artist Pollock's (1912-1956) dripping color and splashing pigment ("Fig. 2"). From the perspective of Pollock's painting methods, the "narrative clues brought about by this dripping, throwing and splashing have never been completely abandoned. But it seems to be messy in order to let go of the touching melodies and


the indescribable feeling and emotional nature".[4] Here, "What distinguishes metaphors is the novelty and instability". In the schema of Pollock's works, the interweaving and superposition of line-like shapes present intense emotions, real confusion, and inner struggle. Bart's fable schema thinks that the first layer of the image is informative and referential, and the second layer is symbolic. Although the works of both artists include "action painting", Tan Ping's paintings show more traces of thinking, internalized faints, and the construction of perception.

Fig. 2. The work of American abstract artist Pollock. 


\section{INTERPRETATION OF MEANING AND REALITY OBSERVATION}

In a specific sense, the action process of abstract painting is related to the present moment of the artist. The artist's thought is closely related to the patterns and images in the painting through the body's drawing behavior, and starting from the inner mood of the artist, a metaphorical reference relationship is formed in the scene of the picture. This reference is related to the specific attributes pointed to by the abstract works, and it all depends on how people feel and understand this correlation. Although metaphors come from the creation process and final presentation of abstract paintings on the one hand, they also come from the interaction between the works and the viewer's thoughts. "In any case, everyone who has experience in art works will undoubtedly incorporate this verified place into himself, that is, into his entire selfunderstanding. Only in this kind of self-understanding does this experience make sense to him".[5] Of course, the abstract works of different artists must have a variety of presentation methods in terms of schema features and work forms: among contemporary Chinese abstract artists, Wang Yigang's work seeks schema order through destruction through physical writing; Ma Shuqing focuses more on how to show the invisible time and space in the visual schema; Meng Luding's works sprayed from the center to the circumferential layers, forming a visual schema similar to the visual illusion in the depth of the interior, not only feeling the ideal performance of scientific thinking, but also feeling a mysterious hint. This diversity of the artist's work patterns more fully characterizes the semantic features and linguistic meanings that appear in the visual patterns, and adds an abstract metaphorical meaning.

Another abstract artist Ding Yi's "Appearance of Crosses 1991-7" and many other works ("Fig. 3") have been deeply influenced by Mondrian, presenting symbolic features and Opple-like interlacing techniques, using continuous and interwoven lines to create a series of works. The visual pattern of this abstract painting is finely arranged and highly rigorous. The entire network structure based on the cross-shaped symbol appears to be many colored grids and lines on the surface, and under the explicit visual logic, it blends various possible schema organization and meaning interpretation. Through the tight graphic symbols, the seemingly ambiguous multiple narrative structure and rational thinking hidden in it are presented, and various visual associations and imaginations are associated. Different cross-shaped schemas also imply different dialogue relationships and philosophical metaphors. This metaphor has both silent appearance and rigorous construction. Although after 2000, "Appearance of Crosses" series of works have more rich changes and organizational logic in the schema, and the picture space also shows more richness and structural levels due to the changing rules, it can still be summarized as the continuous repetition of simple cross-shaped elements. In this seemingly single but richly constructed schema (See "Fig. 4"), Ding Yi insisted on using simple schema elements to construct a large number of conceptual fragments, which connect the artist's inner mapping. Through the time deduction of the cross-shaped series and the persistence of reason, it demonstrates its inner philosophical understanding.

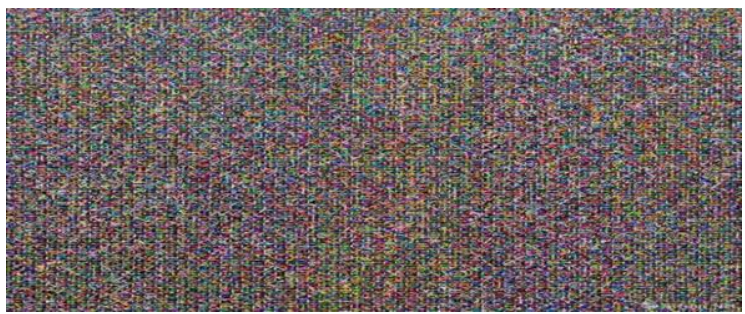

Fig. 3. Chinese abstract artist Ding Yi's "Appearance of Crosses" series of works 1.

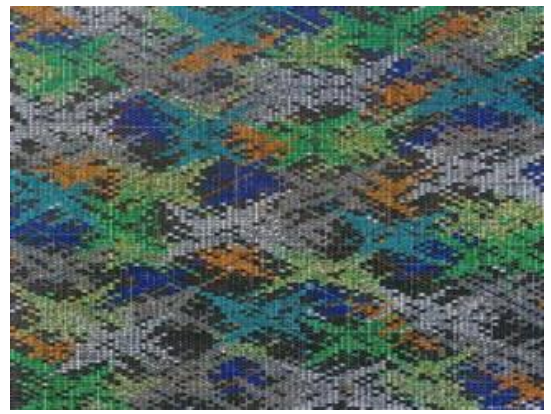

Fig. 4. Appearance of Crosses 2017-6.

"The characteristic of art is that art is a language with all possible levels of understanding".[6] For Goodman, the viewing and understanding of abstract paintings is also a "schematic interpretation of the characteristics of images obtained metaphorically". In this process, both the atmosphere presented by the painting as a whole and the details in the picture refer to the whole as one with the work. The abstraction of an artist's work of art has never been achieved overnight, and often requires a long creative process. The German artist Richter's mid-term paintings have changed the image from visualization to the appearance and obliteration, and have undergone many changes. In the photorealistic painting of the early 1960s, Richter put more photographic features into his paintings as much as possible. In the 1970s, Richter began to randomly choose unintelligibility, visual fragments and external tones and scenes as various heterogeneous graphical elements in the picture, used seemingly irrational presentation methods to transform the visual experience into a specific form of intention and then through the destruction and covering of tools and paints, formed new patterns and constructed meaning. Richter mapped 
the actual observation and formal thinking through the destruction of the picture, the change of color and the overlap, and provided people with a new viewing method and method perspective ("Fig. 5"). The logic of art history is often based on the continuous replacement of methodology, rather than simple image replacement.
In the process of exploring the language of art, Richter continued to question and practice until his later works showed a highly generalized abstract schema, which was different from the previous breaking and covering, tending to reason and order, reflecting the typical characteristics of digital images in the digital age.


Fig. 5. Works by German artist Gerhard Richter.

\section{IMAGE REFERENCE AND MULTIPLE PERCEPTION}

The unique visual schema of abstract paintings comes from the creative process of the artist's different thinking on the one hand, and on the other hand is similar to the release and free expression of lines and colors in early childhood painting. Different creative methods have formed different image schemas, different image personalities, various meaning structures as well as a rich artistic ecology. "As for line drawings, oil paintings and other means of reproduction, all they can do is not simply translating some formed ideas into visible schemas, but also providing help in solving certain problems".[7] Although abstract painting abandons the reproduction of concrete objects, and some even hides and covers multiple visual schemas, it often presents more accurate and contagious language content and thought shapes than concrete ones. Tan Ping's paintings become interrelated series of works through recognizable objects and analyzable types in the picture, which establishes a clearer schema and logic between specific visible objects and visual language, extending the horizon and meaning tolerance of abstract painting. "The metaphor is most effective when the transferred schema creates new and compelling organizations, rather than just re-marking the old schema".[8] It is precisely because of the visual symbols, whether explicit or implicit, or silent or high-profile visual elements presented in abstract paintings, that provide people with a way to understand the work.

In the abstract context, the colors, lines, clumps, and graphics in the visual elements can be read and understood through the visual schema formed by the formal sequence, and even complement the meaning and visual metaphors of the work, presenting the changing qualities of artistic image language.
Moreover, this language interpretation of the work and the image of the work itself can't be completely equivalent. "All works of art must be tracked and understood according to their ideas or their structure in order to become an object of artistic perception. To this end, it requires not only intuition and imagination, but also reflection".[9] It all depends on how people understand this correlation. When people ask how a seemingly silent Roscoe's work shows its emotions, the morphological relationship in the visual image is revealed and perceived from the image medium of subtle observation and reference. The form of the image in the picture is not just the image itself, but is perceived through the content presented in the image and the current viewing psychology. It enriches reality perception with various special perceptions, and through the medium of canvas, it appears in the surface form and the things and matters associated with it.

\section{CONCLUSION}

"We can think of art as a search for new languages, new ways of constructing reality and new means of redefining ourselves. It is a language embedded in form and behavior, text and structure".[10] In abstract painting, the construction of visual images is often accompanied by the formation of meaning and the construction of metaphors. In the construction of multiple levels and contents, the explicit visual schema becomes a direct narrator of meaning. Beneath the surface image, the hidden or covered form elements of the painting process or the hidden elements are also viewed to reflect the self-referential meaning of abstract painting itself. Different viewers form different meanings. No matter what kind of different narrative content the abstract schema is understood as, the existence of this difference is allowed. After all, in understanding the same artistic object, there is inevitably a personal difference between the 
interpretation of the dominant elements and the interpretation of the hidden elements in the picture. Of course, to fully understand an abstract painting, people should not only focus on the way the abstract painting itself is presented, or only the visual schema as a specific artistic medium, but also the process behind the abstraction and the artist's motivation for painting What caused the abstract features people are facing now? How does the work show the artist's creative intention hidden in the work? How does the meaning of the works correspond to the ways and philosophical methods of artistic expression? Although abstract painting doesn't reproduce any specific things, it is still possible to construct a rational meaning through visual patterns presented in abstract paintings, the artist's creative background and the creative process and traces displayed in the picture. After all, most viewers' interpretation of the visual logic in abstract painting is not only sensible but also knowable - Although the degree of sensible and knowable varies. Here, the silent or looming visual schema creates an appropriate way of appearance and the preparation conditions for selfreferentiality for the representation of abstract paintings or potential metaphors.

\section{References}

[1] [Germany] Martin Zell: "Aesthetics of Appearance", translated by Yang Zhen, China Social Sciences Press, p. 200. (in Chinese)

[2] [England] Ascot: "The Future Is Now: Art, Technology and Consciousness", edited by Yuan Xiaoying; translated by Ren Aifan and Zhou Ling. Beijing: Jincheng Publishing House, p. 111. (in Chinese)

[3] [US] Nelson Goodman, "The Voice of Art — The Road to Symbol Theory", translated by Peng Feng; Peking University Press, p. 58. (in Chinese)

[4] [US] Nelson Goodman, "The Voice of Art — The Road to Symbol Theory", translated by Peng Feng; Peking University Press, p. 74. (in Chinese)

[5] [Germany] Hans-Georg Gadamer: "Truth and Method", translated by Hong Handing. Shanghai Translation Publishing House, p. 5. (in Chinese)

[6] [Germany] Biemel, "Philosophical Analysis of Contemporary Art", translated by Sun Zhouxing, Li Yan. Beijing: Commercial Press, p. 278. (in Chinese)

[7] [US] Rudolf Arnheim: "Visual Thinking" Translated by Teng Shouyao. Sichuan People's Publishing House, p. 170. (in Chinese)

[8] [US] Nelson Goodman, "The Voice of Art — The Road to Symbol Theory", translated by Peng Feng. Peking University Press, p. 63. (in Chinese)

[9] [Germany] Martin Zell: "Aesthetics of Appearance", translated by Yang Zhen. China Social Sciences Press, p. 107. (in Chinese)

[10] [England] Ascot: "The Future Is Now: Art, Technology and Consciousness", edited by Yuan Xiaoying; translated by Ren Aifan and Zhou Ling. Beijing: Jincheng Publishing House, p. 167. (in Chinese) 